Preprint

UCRL-JC-147032

\title{
High-Resolution Broadband Spectral Interferometry
}

\author{
D.J. Erskine, J. Edelstein
}

This article was submitted to SPIEs Astronomical Telescopes and Instrumentation, Waikoloa, HI, August 22-28, 2002

\section{August 9, 2002}

U.S. Department of Energy

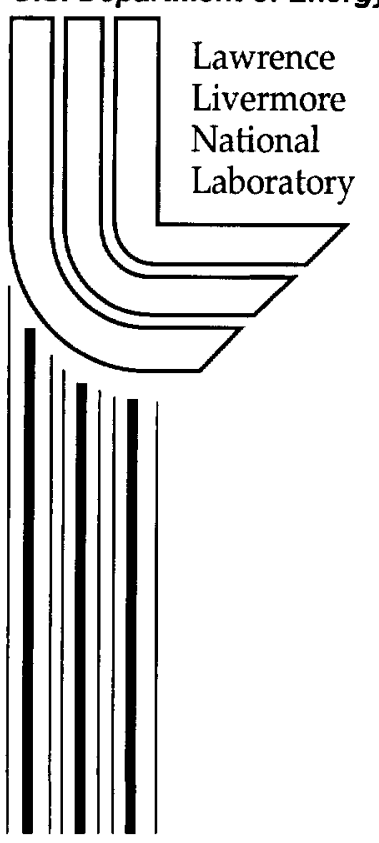




\section{DISCLAIMER}

This document was prepared as an account of work sponsored by an agency of the United States Government. Neither the United States Government nor the University of California nor any of their employees, makes any warranty, express or implied, or assumes any legal liability or responsibility for the accuracy, completeness, or usefulness of any information, apparatus, product, or process disclosed, or represents that its use would not infringe privately owned rights. Reference herein to any specific commercial product, process, or service by trade name, trademark, manufacturer, or otherwise, does not necessarily constitute or imply its endorsement, recommendation, or favoring by the United States Government or the University of California. The views and opinions of authors expressed herein do not necessarily state or reflect those of the United States Government or the University of California, and shall not be used for advertising or product endorsement purposes.

This is a preprint of a paper intended for publication in a journal or proceedings. Since changes may be made before publication, this preprint is made available with the understanding that it will not be cited or reproduced without the permission of the author.

This work was performed under the auspices of the United States Department of Energy by the University of California, Lawrence Livermore National Laboratory under contract No. W-7405-Eng-48.

This report has been reproduced directly from the best available copy.

Available electronically at http://www.doc.gov/bridge

Available for a processing fee to U.S. Department of Energy

And its contractors in paper from

U.S. Department of Energy

Office of Scientific and Technical Information

P.O. Box 62

Oak Ridge, TN 37831-0062

Telephone: (865) 576-8401

Facsimile: (865) 576-5728

E-mail: reports@adonis.osti.gov

Available for the sale to the public from

U.S. Department of Commerce

National Technical Information Service

5285 Port Royal Road

Springfield, VA 22161

Telephone: (800) 553-6847

Facsimile: (703) 605-6900

E-mail: orders@ntis.fedworld.gov

Online ordering: http://www.ntis.gov/ordering.htm

OR

Lawrence Livermore National Laboratory

Technical Information Department's Digital Library

http://www.llnl.gov/tid/Library.html 


\title{
High- Resolution Broadband Spectral Interferometry
}

\author{
David J. Erskine ${ }^{a}$ and Jerry Edelstein ${ }^{b}$ \\ ${ }^{a}$ Lawrence Livermore Nat. Lab., 7000 East Ave, Livermore, CA 94550 \\ ${ }^{b}$ Space Sciences Lab. at Univ. of Calif., Berkeley, CA 94720-7450
}

\begin{abstract}
We demonstrate solar spectra from a novel interferometric method for compact broadband high-resolution spectroscopy. The spectral interferometer (SI) is a hybrid instrument that uses a spectrometer to externally disperse the output of a fixed-delay interferometer. It also has been called an externally dispersed interferometer (EDI). The interferometer can be used with linear spectrometers for imaging spectroscopy or with echelle spectrometers for very broad-band coverage. EDI's heterodyning technique enhances the spectrometer's response to high spectral-density features, increasing the effective resolution by factors of several while retaining its bandwidth. The method is extremely robust to instrumental insults such as focal spot size or displacement. The EDI uses no moving parts, such as purely interferometric FTS spectrometers, and can cover a much wider simultaneous bandpass than other internally dispersed interferometers (e.g. HHS or SHS).
\end{abstract}

Keywords: Externally Dispersed Interferometer, Spectral Interferometry, Fourier Transform Spectrometer, High Resolution Spectroscopy, Radial Velocimetry, Doppler Planet Search

\section{INTRODUCTION}

We describe a hybrid dispersive/interferometric technique that can achieve resolutions $(R=\lambda / \Delta \lambda)$ in the $\mathrm{R}=50,000$ regime at competitive photon-limited signal to noise ratio as a grating, and yet is only $\sim 1 \mathrm{~m}$ in length. The technique is called Spectral Interferometry (SI) or Externally Dispersed Interferometry (EDI) and has been developed for the Doppler Planet Search by Lawrence Livermore National Laboratory beginning in $1997 .{ }^{1-6}$ The same apparatus used for radial velocity measurements can be used for high resolution spectroscopy over a very wide bandwidth, limited only by the bandwidth of an external spectrograph. One-dimensional imaging spectroscopy is possible. In comparison with other high resolution techniques achieving at least R $50 \mathrm{k}$, the EDI has significant advantages. Purely dispersive spectrographs are expensive, large ${ }^{7,8}$ ( $5 \mathrm{~m}$ scale), and sensitive to beam profile. And purely interferometric instruments such as the Fourier Transform Spectrometer (FTS) have a photon-limited signal to noise ratio (s/n) 2 orders of magnitude poorer.

\section{APPARATUS}

The EDI is the series combination of an undispersed fixed delay interferometer and an external grating spectrograph. Figure 1 shows the schematic of the EDI and a photograph of the interferometer portion, which is very compact. Fringes formed at the interferometer mirror plane are imaged to the slit plane by a lens. When dispersed by the grating spectrograph, this forms a fringing spectrum detected by a CCD. One of the interferometer mirrors is mounted on a PZT transducer so that the interferometer delay can be stepped (dithered) in small increments as a piston. Ideally, the phase stepping uses either 3 exposures at $\lambda / 3$ or 4 exposures at $\lambda / 4$.

\subsubsection{Linear vs Echelle grating formats}

The EDI can use either linear or 2-d cross-dispersed echelle spectrographs, and this choice affects the data taking mode. The EDI has been demonstrated in both configurations: with a linear grating at $R=20 \mathrm{k}$ for sunlight ${ }^{3}$ and $R=6 \mathrm{k}$ for stellar measurements ${ }^{4}$ and with the Lick Observatory $\mathrm{R}=60 \mathrm{k}$ echelle grating spectrograph for recent stellar and sunlight measurements. The advantage of the echelle spectrograph is a very much wider bandwidth at high resolution than a linear grating.

Further author information:

D.J.E.: E-mail: erskine1@llnl.gov, Telephone: 19254229545

J.E.: E-mail: jerrye@ssl.berkeley.edu, Telephone: 15106420599 
With the linear grating spectrograph the fringe phase is varied linearly along the slit length by tilting an interferometer mirror. In this case piston phase stepping is not required, since all fringe phases are recorded in a single exposure, splayed transversely to the dispersion axis. However, the use of phase stepping is recommended because it reduces the instrument noise. It discriminates against fixed pattern noise that can mimic as spatial fringes- only true fringes will vary versus phase step synchronously.

The use of the echelle format, with its very short slit length, precludes splaying the fringe phase spatially. Instead the phase (interferometer delay $\tau$ ) is made uniform over the beam. Analyzing data taken in this mode required our development of appropriate software.

\subsubsection{Spectral Comb}

The EDI approach is to use the frequency response $T(\nu)$ of an undispersed Michelson interferometer as the key element to discriminate behavior in the input spectrum. Let the dispersion axis of the external disperser be described by frequency and wavelength $\nu \equiv 1 / \lambda$, and be horizontal. The units of $\nu$ are $\mathrm{cm}^{-1}$. Let the vertical or $y$-axis of the CCD record be along the length of the entrance slit of the external spectrograph. The optical path length difference between the two interferometer arms is called the delay $(\tau)$, in units of $\mathrm{cm}$. Then the transmission function $T(\nu, y)$ of one output arm is

$$
T(\nu, y)=\frac{1}{2}\left[1+\alpha \cos \left(2 \pi \tau \nu+\phi_{y}\right)\right],
$$

where $\phi_{y}$ is the interferometer phase described further below, and $\alpha$ is the interferometer fringe visibility, which is unity for the ideal situation where the intensities of the two interfered arms are matched.

Figure 2 compares the EDI technique with the conventional grating-only method, and with another interferometer/grating hybrid technique called the Heterodyning Holographic Spectroscopy (HHS). ${ }^{10-12}$ The latter differs from the EDI by incorporating a grating internal to the interferometer cavity. Under white light illumination $T(\nu, y)$ creates a very periodic sinusoidal spectral comb, as shown in panel b). This extends over all wavelengths with the same periodicity $\Delta \nu=1 / \tau$. In contrast the HHS has a fringe spacing which is strongly frequency dependent, because its grating changes the angle at which the beams internal to the interferometer combine. This has the effect of limiting the bandwidth, as can be seen by the limited extent of the diamond-like fringe pattern in panel $\mathrm{c}$ ).

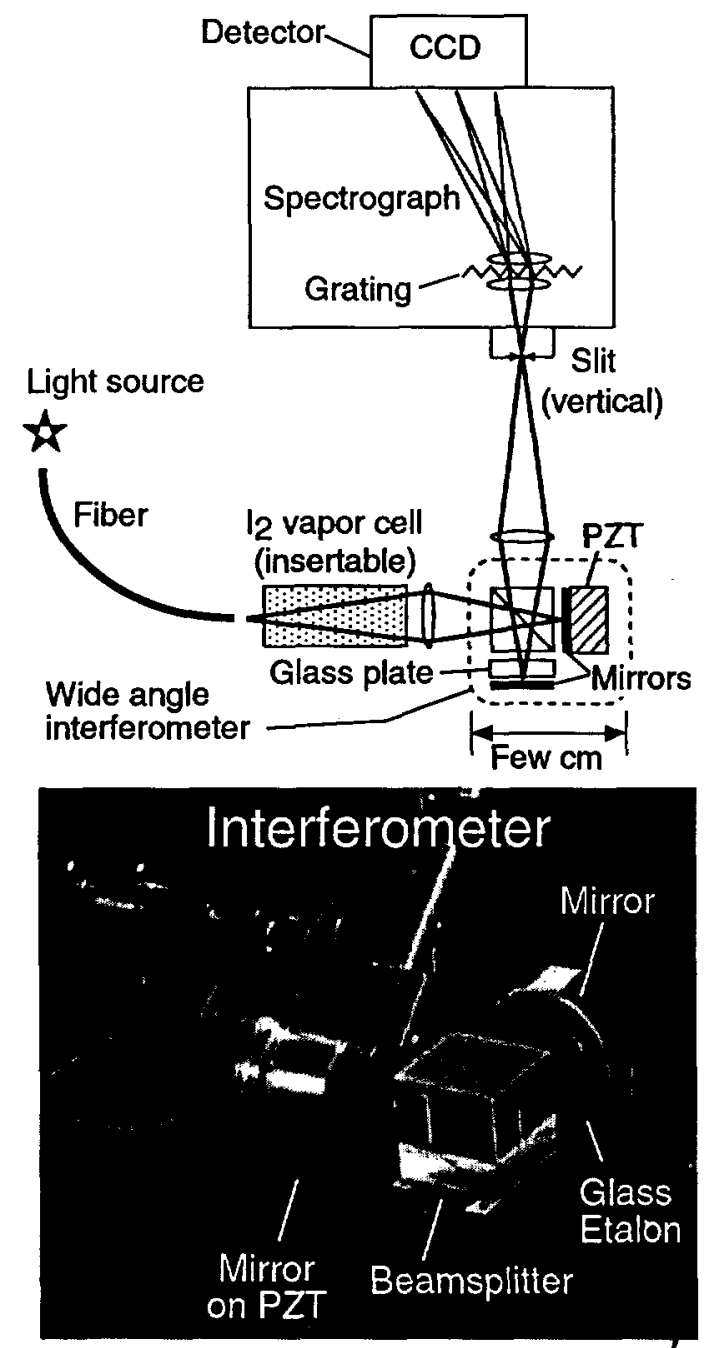

Figure 1. Schematic of the EDI and photo of interferometer subassembly. Sunlight from a roof-mounted heliostat conducted through fiber leads to an iodine vapor cell which provides reference spectrum. Wide-angle interferometer ${ }^{9}$ with $11 \mathrm{~mm}$ fixed delayy created by glass plate (etalon) imprints fringes on beam at spectrograph slit, creating a fringing spectrum at CCD. Jobin-Yvon HR640 grating spectrograph (0.7 m length) with $R \sim 20000$ disperses light. Lens images slit plane to the mirror plane where the fringes are located, and on to the source plane, which could be a star image in non-fiber system. The etalon shifts the virtual image of one of the mirrors closer to the beamsplitter, so that after they are longitudinally superimposed the spatial delay is zero but the temporal delay is nonzero. This produces the angle independence. PZT transducer increments interferometer delay in 3 or 4 steps of $\lambda / 3$ or $\lambda / 4$. This phase stepping procedure isolates fringing from nonfringing spectral components. 

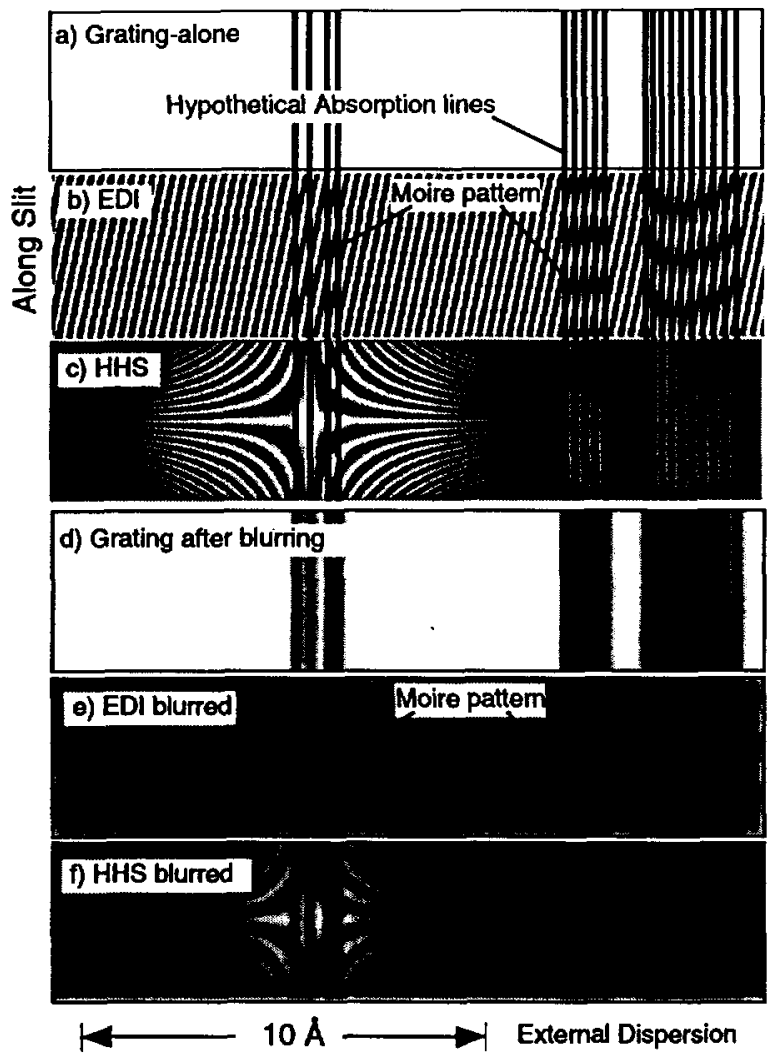

Figure 2. Comparison of three different spectroscopy techniques. Spectrograph blurring is neglected in a), b), c) but imposed in $\mathrm{d}$ ), e), and $\mathrm{f}$ ). In a), d) is a conventional grating spectrograph. Blurring causes loss of high spatial frequency information. In b), e) the EDI a uniform grid created by the interferometer overlays the spectrum. This creates Moire fringes that encode high spatial frequency information about the spectrum. These survive the blurring of the spectrograph slit, as seen in e). Moiré fringes can be created over the full bandwidth of the external spectrograph. In contrast, the Heterodyning Holographic Spectrograph $^{10-12}$ (HHS) c), f) uses a grating internal to the interferometer. Consequently the transverse fringe spacing diverges around a fixed wavelength, limiting the bandwidth.
For the EDI the periodic spectral comb interacts with the input spectrum to form Moiré fringes. These carry information about the spectrum that can survive blurring imposed by the slit width or focal spot diameter of the spectrograph. The effect of blurring can be seen simulated in panel e). The paramount purpose of the EDI data taking procedure and analysis is to accurately measure the shape and phase of this Moiré pattern. Measuring the underlying interferometer spectral comb is not strictly necessary. In principle the EDI technique can be applied even if the spectral comb is completely blurred or beyond the Nyquist resolving limit of the CCD. However, in practice it is useful to at least partially detect the interferometer comb since it can assist in determining phase step size in a noisy environment, and indicate location of nonuniformities in the spectrograph/CCD.

\subsubsection{Phase Stepping Modes}

Generally speaking, in order to accurately determine a fringe phase and amplitude one needs to sample the output intensity at 3 or more instances where the interferometer delay is dithered $\Delta \tau$ slightly about its average value. The dither is equivalently described by an interferometer phase

$$
\phi_{y}=2 \pi(\Delta \tau / \lambda) .
$$

This process is called phase stepping. A good general discussion is by Greivenkamp \& Bruning (1992) and references therein. Here phase stepping can be implemented by two independent means, a) versus time in multiple exposures, by moving the entire interferometer mirror as a piston using the PZT transducer in steps of size $\Delta \phi_{p}$; and b) sampling all phases simultaneously in each exposure, by tilting one interferometer mirror relative to the other to vary the phase linearly vs $y$. (The transverse and dispersion axes are $y$ and x.) This creates "phase-slanted" or "splayed" fringes having spatial period $P_{y}$ along the slit length. The combination of these two dithering modes is

$$
\phi_{y}=2 \pi\left(y / P_{y}\right)+n \Delta \phi_{p}
$$

where $n$ indexes the piston exposures.

Phase-slanted mode. In the early use of the EDI the phase-slanted method was used predominantly. This was because of its practical advantage of making the Moiré fringe visibility immediately apparent from the CCD image. Typically 4 to 6 fringe periods were created along the $y$-extent of the beam, which was $\sim 60$ pixels tall. Under white light illumination this creates a fringing spectrum having slanted fringes across 
the whole bandwidth, as seen in Fig. $2 \mathrm{~b}$ and in data Fig. 6a.

Phase-uniform mode. Alternatively, the fringe phase can be made uniform across the beam by aligning the interferometer mirrors with each other. Then the spatial extent of the fringe $P_{y}$ expands to infinity. This "phase-uniform" mode of taking data has the advantage of not requiring an imaging spectrograph, and not being sensitive to blurring along the $y$-axis due to astigmatism in the spectrograph optics. Secondly, it allows the use of the EDI technique with echelle grating spectrographs, which have short slits in order to prevent the grating orders from overlapping. With the small star image presented to the echelle slit, it is easy to arrange the interferometer phase to be uniform across the whole beam. We have recently demonstrated this mode of taking data on the Lick echelle spectrograph on starlight and sunlight (Figs. 7 and 8 below).

In the phase-uniform mode the interferometer spectral comb has vertical fringes that depend only on $\nu$. Note that mathematically a single phase-slanted exposure can be considered a set lineouts along the dispersion axis, with each lineout being a phase-uniform exposure having a different phase, evenly distributed around the phase circle.

By combining the $\cdot 2$ means of dithering phase we have 3 modes of taking data:

1. Phase-slanted in a single exposure.

2. Phase-slanted in multiple exposures that are piston stepped.

3. Phase-uniform in multiple exposures that are piston stepped.

In method \#3 a minimum of three piston exposures are needed to fully determine the Moiré component of the signal. In method \#1, although a single phase-slanted exposure is sufficient to measure the Moiré pattern, it is susceptible to fixed pattern errors that mimic the appearance of fringes. These could be CCD pixel gain variations or parasitic interference patterns from other optical surfaces. Such fixed pattern noise can produce a significantly large Doppler velocity error. Hence taking multiple exposures in the phaseslanted mode is recommended if the EDI is used for 1 $\mathrm{m} / \mathrm{s}$ scale radial velocity measurements.

\section{THEORY OF OPERATION}

\subsubsection{Normalized Interferometer Spectral} Comb

The starting point for the analysis is the ideal interferometer spectral comb normalized to average value of unity so that the total number of photons at the detector is the same between the EDI and conventional (grating-only) techniques.

$$
T^{\prime}(\nu, y)=1+\cos \left(2 \pi \tau \nu+\phi_{y}\right)
$$

This facilitates comparison of photon limited performance, because the photon noise is due to the continuum, which will be the same in both methods. Also, this separates the issues of instrument loss from instrument response.

In the simple Michelson design used for the prototype, only one output was used and hence the loss would be $50 \%$. However, a Mach-Zehnder interferometer design would allow both output arms to be directed through the spectrograph and detected on adjacent CCD pixels, ideally producing no net loss. This can also be done with interferometers using polarization to encode the two complementary outputs that travel generally in the same direction, which are eventually split to different pixels at the CCD. On an echelle the space between orders could detect the complementary output. These lossless configurations would produce Equation 4.

\subsubsection{Conventional Spectroscopy}

Let the intrinsic input spectrum be denoted $S_{0}(\nu)$, and the point spread function (blurring response of the spectrograph for a pure frequency) denoted $\operatorname{PSF}(\nu)$. Then in ordinary (purely dispersive) spectroscopy the detected signal is the convolution

$$
S_{\text {ord }}(\nu)=S_{0}(\nu) \otimes P S F(\nu)
$$

The blurring action (convolution) is most conveniently expressed in the Fourier-space, as

$$
s_{\text {ord }}(\rho)=s_{0}(\rho) p s f(\rho),
$$

where the convolution operation becomes the ordinary multiplication. Let the lower case denotes the Fourier transform of a given function, i.e. ps $f(\rho)$ is the Fourier transform of $P S F(\nu)$.

This function $s(\rho)$ could be called the distribution of feature-densities. High feature density $\rho$ corresponds to narrow and detailed structures in $S(\nu)$. While $s(\rho)$ has significant value over a range of $\rho$ from 0 to $3 \mathrm{~cm}$, the most important region for Doppler velocimetry is near $\rho \sim 1 \mathrm{~cm}$, i.e. 1 feature per $\mathrm{cm}^{-1}$. And for analyzing the asymmetry in a lineshape, the region 1.5 to $4 \mathrm{~cm}$ is most important. 


\subsubsection{EDI Spectroscopy}

The passage of light through the interferometer imprints (multiplies) the spectral comb $T(\nu)$ against the spectrum prior to the blurring action of the external grating spectrograph. Hence the EDI detected signal is

$$
S_{E D I}(\nu)=\left[S_{0}(\nu) T^{\prime}(\nu)\right] \otimes P S F(\nu),
$$

which becomes a sum of the ordinary spectrum plus the new fringing components.

$$
\begin{aligned}
S_{E D I}(\nu)= & S_{\text {ord }}(\nu)+\frac{1}{2}\left[S_{0}(\nu) e^{i \phi_{\nu}} e^{i 2 \pi \tau \nu}+\right. \\
& \left.S_{0}(\nu) e^{-i \phi_{\nu}} e^{-i 2 \pi \tau \nu}\right] \otimes P S F(\nu) .
\end{aligned}
$$

In Fourier-space this is

$$
\begin{aligned}
s_{e d i}(\rho)= & s_{\text {ord }}(\rho)+\frac{1}{2} e^{i \phi_{y}} s_{0}(\rho+\tau) p s f(\rho)+ \\
& \frac{1}{2} e^{-i \phi_{v}} s_{0}(\rho-\tau) p s f(\rho),
\end{aligned}
$$

where we used the well known property of Fourier transforms that multiplication by a phasor $e^{i 2 \pi \tau \nu}$ will shift (by amount $\tau$ ) the result in the conjugate Fourier variable $\rho$. The result consists of the ordinary detected signal $s_{\text {ord }}$, plus two fringing terms which counter-rotate in the complex plane versus $\phi_{y}$.

\subsubsection{Conversion to a Vector Spectrum: Whirl}

The purpose of measuring a fringing spectrum at multiple phase outputs $\left(\phi_{y}\right)$ is to isolate one of these two fringing components so that a Doppler effect rotates the signal in one direction only. The single polarity rotation allows a simple geometric (vector-based) interpretation of the phase of the various signal components, not possible in the presence of two counter-rotating versions of each component. The scalar spectrum is converted to a vector spectrum (a complex wave) called a "whirl".

The formation of the complex whirl from scalar but phase-slanted set of fringing data is a kind of phase stepping. Due to limited space, let us only discuss data taking mode \#1: phase-slanted in a single exposure. Data taking mode \#2 is explained in Ref. 6, and mode \#3 will be described in detail in a future publication. Consider the single CCD exposure as a set of lineouts $I(\nu)$ having various $\phi$ depending on $y$. One can combine $I(\nu)$ from different $y$-channels in a linear combination analogous to the 4-bucket algorithm described in Greivenkamp \& Bruning (1992), but using complex weightings. For clarity, let us bin the interferometer outputs into 4 components separated by $\Delta \phi_{y}=90^{\circ}$ intervals, and designate these $S_{\text {edi }}\left(\nu, \phi_{y}\right)$ as $S\left(0^{\circ}\right), S\left(90^{\circ}\right)$ etc. (An equation using 3 components at $120^{\circ}$ separation is similar but less elegant in appearance). Let the whirl (W) be a linear combination of the scalar lineout data, using a prefactor of (1/4) so that the total flux is the same as in a single exposure.

$$
\mathrm{W}(\nu)=\frac{1}{4}\left\{\left[S\left(0^{\circ}\right)-S\left(180^{\circ}\right)\right]+i\left[S\left(90^{\circ}\right)-S\left(270^{\circ}\right)\right]\right\}
$$

\subsubsection{Operational Computation of Whirl}

Equation 10 is useful for formal analysis, but operationally, the whirl is formed from the raw CCD intensity data $I(\nu, y)$ by a different but mathematically equivalent method. This is easy to calculate and uses all the y-channels, not requiring them to be spaced every $90^{\circ}$.

The average period of the spatial fringe along the $y$-axis is first found. Using this period, for each CCD $\nu$-channel the fixed period Fourier amplitude is taken of the intensity profile $I(y)$ by multiplying it against a previously computed sine or cosine wave and then summing over $y$-pixels. Then the resulting cosine and sine Fourier amplitudes are assigned to the real and imaginary parts of $\mathbf{W}$ :

$$
\mathrm{W} \propto \text { Fourier YCosAmp }+i \text { FourierYSinAmp } .
$$

Equation 11 is extremely fast to compute, even on a small computer. Alternatively, $I(y)$ can be fitted to a fixed period sinusoid with the resulting phase and amplitude assigned to $W$ in polar coordinates, then $\mathrm{W}$ is converted to rectangular coordinates.

\subsubsection{Formation of Moiré Fringes}

Now let's return to the formal analysis. Applying the linear combination in Eq. 10 to Eq. 9 we get the whirl in Fourier space

$$
\mathbf{w}(\rho)=\frac{1}{2} s_{0}(\rho+\tau) p s f(\rho) .
$$

This important equation describes the formation of the Moire fringes. These actions are illustrated in Fig. 3. The psf $(\rho)$ of a grating can be modeled as a gaussian peak centered at $\rho=0$, ie. only broad features of the spectrum $S(\nu)$ survive in a conventional spectrograph. In contrast, for the EDI the argument in $s_{0}(\rho+\tau)$ shows that the input spectrum is shifted (heterodyned) in $\rho$ space, and that high detail spectral information therefore survives the blurring imposed by the spectrograph slit that would normally destroy this information. 

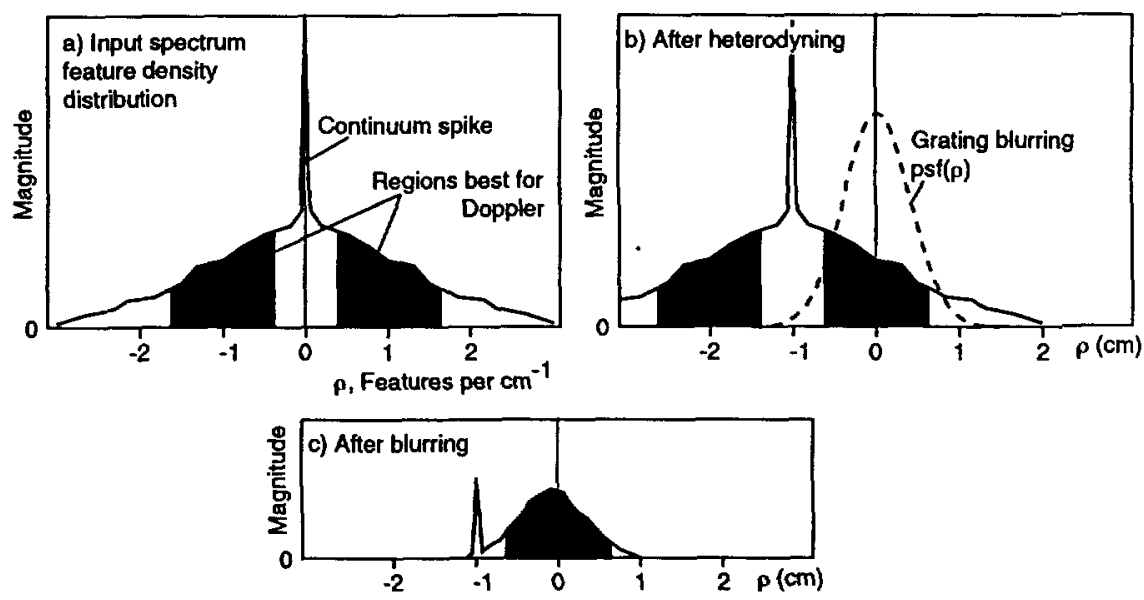

Figure 3. Stages in heterodyning process, in Fourier space. a) The feature-density distribution $s(\rho)$ of input spectrum. The spike at $\rho=0$ corresponds to the continuum. The regions near $\rho \sim \pm 1 \mathrm{~cm}$ hold the most Doppler information and these are blackened for labeling. b) After passage through the interferometer, which multiplies sinusoidal $T(\nu)$ function against $S(\nu), s(\rho)$ shifts by $\Delta \rho=\tau$. This places a blackened regions near the origin where it better survives the grating blurring $p s f(\rho)$ (dashed peak). The other, up-heterodyned blackened region does not survive. c) The continuum spike remnant, now shifted to $\rho \sim-1 \mathrm{~cm}$, corresponds to the periodic sinusoidal comb seen in the CCD data (Fig. $6 \mathrm{a}$ ). The data near the origin expresses the Moiré fringe.

\subsubsection{Nonfringing component}

The ordinary (nonfringing) spectrum can be obtained from the set of fringing spectra by vector addition, so that the fringing terms cancel.

$$
S(\nu)=\frac{1}{4}\left\{S\left(0^{\circ}\right)+S\left(180^{\circ}\right)+S\left(90^{\circ}\right)+S\left(270^{\circ}\right)\right\}
$$

Alternatively but less accurately, the nonfringing spectrum can be obtained from a single exposure by simply summing over all $y$-pixels for each $\nu$-channel of the CCD data.

\subsection{Effective Instrument Response}

Figure 3 shows behavior vs $\rho$ of the output, not input signal. The heterodyning changes the $\rho$ between input and output. For comparing techniques it is useful to describe the effective instrument response by the $\rho$ of the input. Hence we change variables $\rho \rightarrow(\rho-\tau)$. Then Eq. 12 becomes

$$
\mathbf{w}(\rho-\tau)=\frac{1}{2} s_{0}(\rho) p s f(\rho-\tau)
$$

This shows that the original grating response psf $(\rho)$ has been shifted toward higher $\rho$ by an amount $\tau$, and also reduced in amplitude by half. We summarize the effective responses for the two techniques, ordinary and EDI when using the same grating:

$$
\begin{array}{r}
p s f_{o r d}=p s f_{0}(\rho), \\
p s f_{e d i}=\frac{1}{2} \alpha p s f_{0}(\rho-\tau),
\end{array}
$$

where $\alpha$ is the interferometer visibility, which is unity in the ideal case, and $p s f_{0}(\rho)$ is the grating response.

Figure 4a illustrates how heterodyning shifts the grating PSF peak in $\rho$-space, and panel b) illustrates the corresponding PSF in $\nu$-space. By choosing $\tau \sim 1 \mathrm{~cm}$ the EDI psf( $\rho)$ peak can be positioned over the most important region for Doppler velocimetry and other science. Other choices of $\tau$ are possible. The optimum value depends on the narrowness of the features needed to be resolved. 

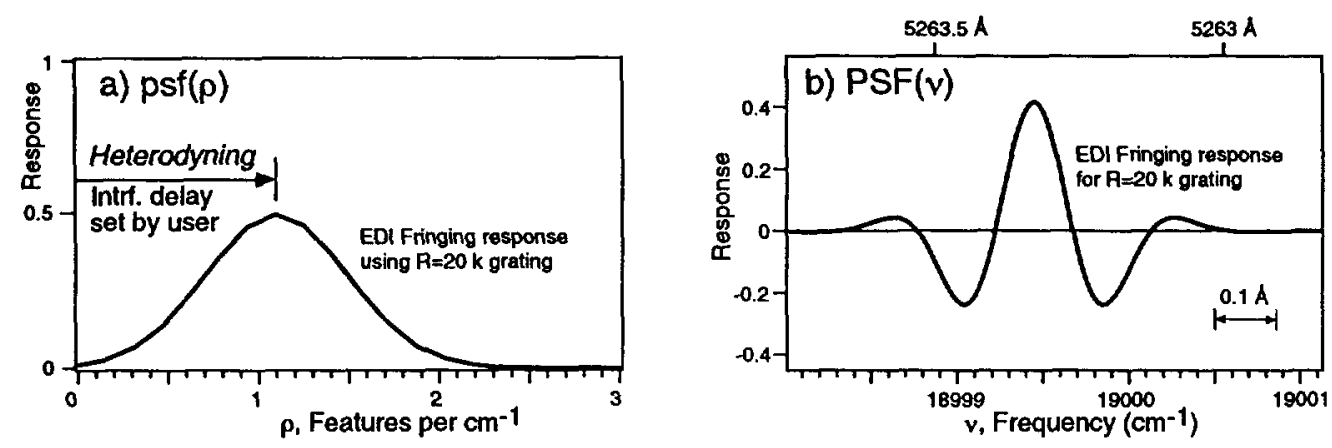

Figure 4. The EDI instrument response concerning just the fringing component. The responses are plotted in both a) Fourier space as $p s f(\rho)$, and b) frequency space as $P S F(\nu)$. The frequency $\nu=1 / \lambda$ is in units of $\mathrm{cm}^{-1}$. The variable $\rho$ is the density of spectral features, in units of features per $\mathrm{cm}^{-1}$. The $p s f(\rho)$ of the grating by itself is modeled as a gaussian peak of unity height centered at $\rho=0$ (not shown). The heterodyning action creates a new peak (shown) which has same shape but half the height and translated by amount $\tau$ toward higher $\rho$, where $\tau$ is the interferometer delay, chosen by user. b) The $\operatorname{PSF}(\nu)$ corresponding to the heterodyned peak is the Mexican sombrero-like function, obtained from $p s f(\rho)$ by Fourier transform. This is the fringing portion of the EDI PSF.

The corresponding $P S F(\nu)$ function has a Mexican sombrero shape. A key point is that the shape of the inner peak of the "hat" has a very accurately defined shape, that is set by the interferometer rather than the grating. It can be shown that the sombrero shape is a sinusoid enveloped (multiplied) by the broader gaussian shape of the grating alone.

\subsection{Reversal of Heterodyning}

The heterodyning effect that occurs optically in the EDI optics can be reversed numerically during data analysis to reconstruct a portion of the input spectrum. Since the optical heterodyning was a downshifting that lowered $\rho$, then this reconstructed portion will contain higher $\rho$ features than what would normally be resolved by the spectrograph without the interferometer. This is analogous to the treble channel of a stereo loudspeaker. This "treble" channel can be combined with the "bass" channel to provide a more full bodied response, where the bass channel is the ordinary nonfringing spectrum Eq. 13. The value of $\tau$ is easily found in the data by inspecting the Fourier transformed whirl and looking for a spike.

The procedure to reverse Eq. 14 is to take the whirl $W(\nu)$, Fourier transform it from $\nu$-space to $\rho$-space, translated it by amount $\tau$ toward higher $\rho$, multiply it by $2 / \alpha$, then inverse transform it back to $\nu$-space to form $W^{\prime}(\nu)$. Because we want positive $\rho$ 's to also represent the negative $\rho$ branch, prior to inverse Fourier transformation we copy the positive branch to the negative after taking the complex conjugate. (Equivalently, we can take the real part of $W^{\prime}(\nu)$ and double it.) This forms $S^{\prime}(\nu)$, the treble portion of the output spectrum.

The bass and treble signal components are added to form the composite spectrum. Then an equalization step can be applied to change the relative strength of the $\rho$ components so that the resulting instrument response is smoothly varying vs $\rho$ rather than have a dip in it as shown in Fig. 5. The user can design the output response to be of any shape, gaussian or flat within a range, for example. However, beware that there is no point in overly magnifying a region of $\rho$ which has poor signal to noise ratio, since it will remain having a poor $\mathrm{s} / \mathrm{n}$ ratio. The equalization process does not change the underlying $\mathrm{s} / \mathrm{n}$ ratio, which is set by the unequalized behavior, shown in Fig. 5. Measurements of the same instrument on a known spectrum such as iodine can be used to determine the shape of psf( $\rho)$, which can assist in determining equalization that removes much of the instrument artifacts.

Figure 5 shows the instrument responses of the EDI compared to the conventional grating-only method. Since the purpose here is to suggest photon signal to noise capabilities, the composite response shown here is not the linear sum of bass and treble responses, (which is what would be plotted if the discussion were about 

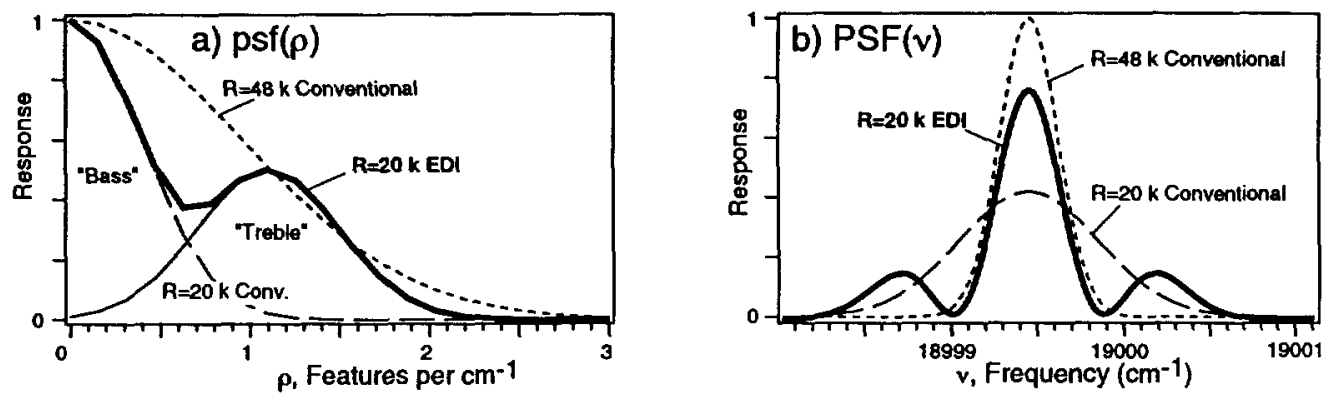

Figure 5. The instrument response of the EDI using $\mathrm{R}=20 \mathrm{k}$ grating (bold) is compared with the conventional method (ie. grating alone, dashed curve) at $\mathrm{R}=48 \mathrm{k}$. a) shows $p s f(\rho)$ in Fourier space, and b) shows $P S F(\nu)$ in frequency space. The $R=48 \mathrm{k}$ and $\mathrm{R}=20 \mathrm{k}$ gratings form broad (dashed) and narrow (long dashed) $p s f(\rho)$ peaks in a), and conversely, narrow (dashed) and wide (long dashed) peaks $P S F(\nu)$ in b). In a) the long dashed peak is the ordinary, nonfringing portion EDI data ("bass"). The EDI also forms a heterodyning peak (thin curve) centered at $1.1 \mathrm{~cm}$ ("treble"). The composite EDI response (bold) is the sum in quadrature of bass and treble responses. In $b$ ) the effective response of the EDI (bold) is the Fourier transform of the bold curve in a). Note that the width the inner peak is similar to the $R=48$ $k$ conventional peak, in spite of using only a $R=20 \mathrm{k}$ grating. The shape of the inner peak is very precise because it is determined by the sinusoidal nature of the interferometer.

the equalization procedure). Instead, the composite $p s f(\rho)$ shown here is the sum in quadrature of the bass and treble components. This is more representative of the net photon limited $\mathrm{s} / \mathrm{n}$ ratio. This is because the treble and bass (fringing and nonfringing) signal components originate from different spatial frequencies in the raw CCD data, so they are statistically independent.

The PSF in frequency space of the Figures was obtained by inverse Fourier transforming the $p s f(\rho)$. Note that the width of the inner peak of the EDI PSF is similar to that of the grating-only technique using a 2.4 times greater resolution, and the height is similar. This is a theoretical explanation of the resolution boosting effect.

\subsection{Experimental Demonstrations of Resolution Boosting}

Figures 6 and 8 show experimental demonstrations of the resolution boosting effect using the EDI on the solar spectrum. The increase in effective resolution is approximately $2.5 \times$. Figure 6 demonstrates it on a linear grating with initial resolution $R=20 \mathrm{k}$, and Fig. 8 demonstrates it on the Lick echelle grating with initial resolution $\mathrm{R}=60 \mathrm{k}$. Figure 7 shows the appearance of the fringing spectra in the echelle format, for the star $\epsilon$ Leo and interferometer delay $1.1 \mathrm{~cm}$. (The interferometer fringes in the $\tau \equiv 3 \mathrm{~cm}$ data is less dramatic because it approaches the CCD Nyquist limit.)

The benefit of adding an interferometer to an existing spectrograph is a factor $\sim 2.5 \times$ boost in the effective resolution while keeping the slit width constant, and hence photon flux constant. Whereas normally to achieve such high resolution the Lick spectrograph would have to use a much smaller slit, which would reduce flux from blurry star images. Alternatively, for Doppler velocimetry where $R=60 \mathrm{k}$ is adequate, one could widen the echelle slit to $R=24 \mathrm{k}$ to increase the flux from a blurry star image, and use the EDI technique to achieve the effective $R=60 \mathrm{k}$ resolution. A further benefit is to widen the utilized bandwidth by switching from the $I_{2}$ cell to a ThAr lamp as spectral fiducial.

\subsection{Multiple Delay EDI}

We have identified how to modify the interferometer so that different parts of the beam can receive different delays, and all of these subbeams can pass through the same external spectrograph. Suppose the delays are chosen to be appropriately distributed, such as $1.1,2.2$ and $3.3 \mathrm{~cm}$, then this greatly extends the resolution of the resulting EDI, as shown in Fig. 9, with minimal decrease in the photon $\mathrm{s} / \mathrm{n}$ ratio. The resolution boost in this particular case is $\sim 10$ times. As the number of delays $M$ increases, the photon $\mathrm{s} / \mathrm{n}$ decreases as $1 / \sqrt{M}$. 

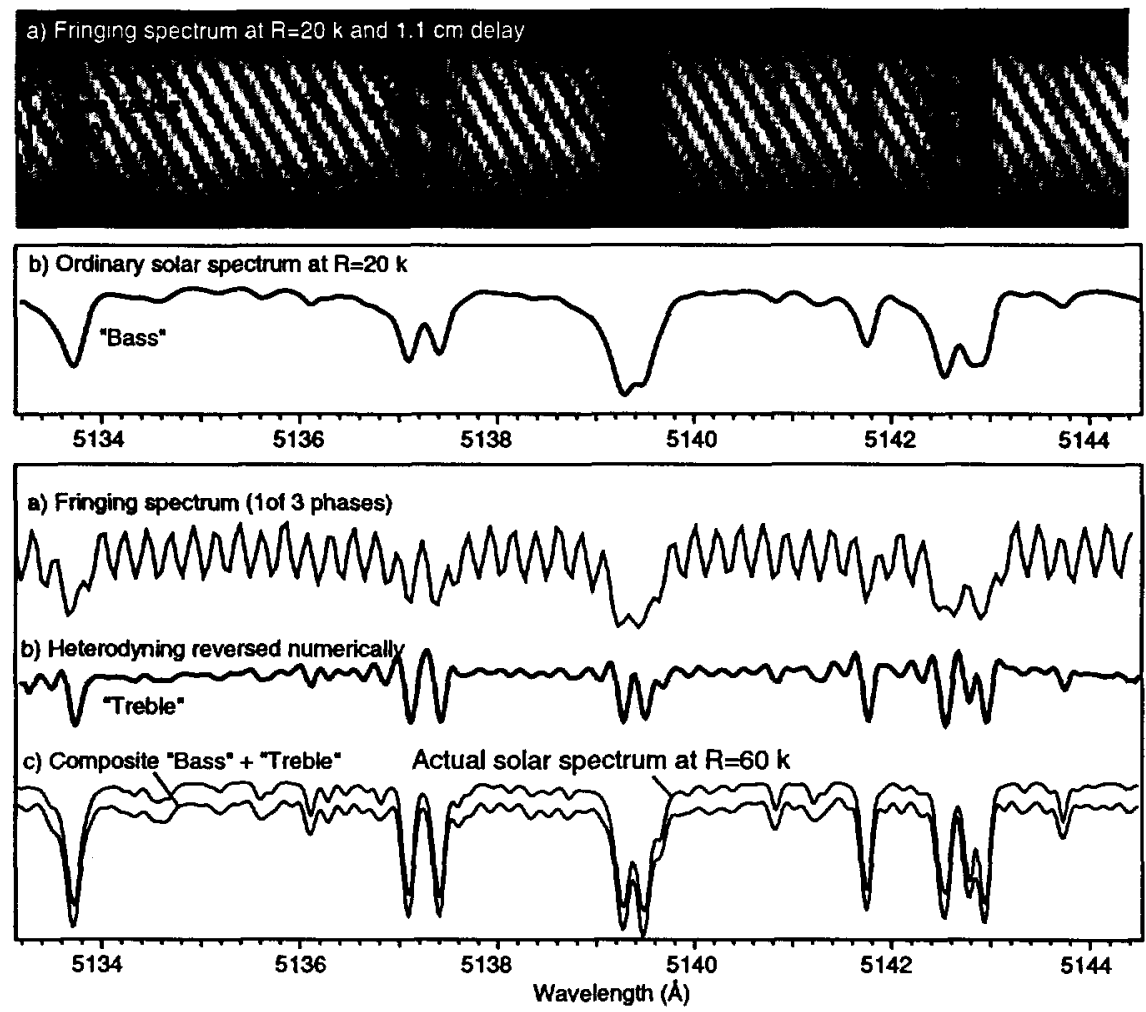

Figure 6. Demonstration of resolution boosting on a $R=20 \mathrm{k}$ linear grating spectrograph and the solar spectrum. a) The fringing spectrum obtained with a fixed $11 \mathrm{~mm}$ delay interferometer in series with the spectrograph. The interferometer phase varies linearly along slit height. Only a subset of full wavelength range is shown. b) The ordinary spectrum ("bass" signal) obtained when the intensity data is averaged along each y-column, destroying fringes. c) A line-out of a) at fixed $\mathrm{Y}$ along dispersion axis. Three or more such lineouts are needed to isolate the fringing from nonfringing components. d) The heterodyning that occurs optically is reversed numerically to form the "treble" signal. e) Composite (lower) of bass and treble signals, after minor equalization to smooth instrument response using iodine calibration spectra. Comparison to Kitt Peak/NOAO FTS solar spectrum (upper) artificially blurred to $R=60 \mathrm{k}$ shows excellent agreement. Data taken June 16, 1998 at LLNL by Erskine with the first EDI prototype.

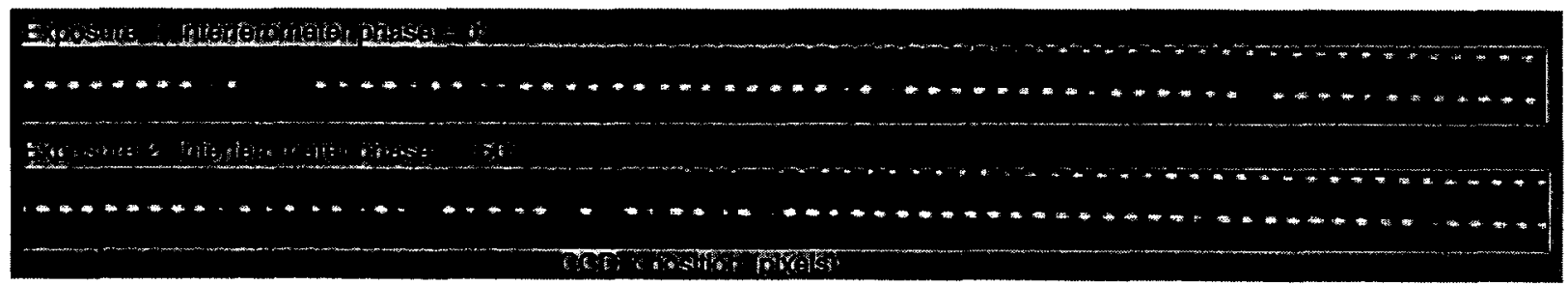

Figure 7. Two expasures of the telluric line order (near $6868 \AA$ ) of star $\epsilon$ Leo taken April 2002 with the Lick echelle spectrograph and a $11 \mathrm{~mm}$ fixed delay interferometer at its slit. The interferometer delay was changed by $150^{\circ}(0.4 \lambda)$ between the two exposures. The spacing of sinusoidal interferometer fringes is $1 /$ delay $=1 / 1.1 \mathrm{~cm}=0.9 \mathrm{~cm}^{-1}$. Note the difference in the Moiré patterns, which manifests interaction between sinusoidal interferometer spectrum and features in the stellar spectrum having similar width as $0.9 \mathrm{~cm}^{-1}$. Three such exposures at minimum are needed to fully isolate the fringing (Moiré) signal from ordinary spectrum. The difference between the phase step exposures isolates the fringing component, the sum isolates the nonfringing. Only one order of about 70 orders spanning from 4000 to $8000 \AA$ is shown. 

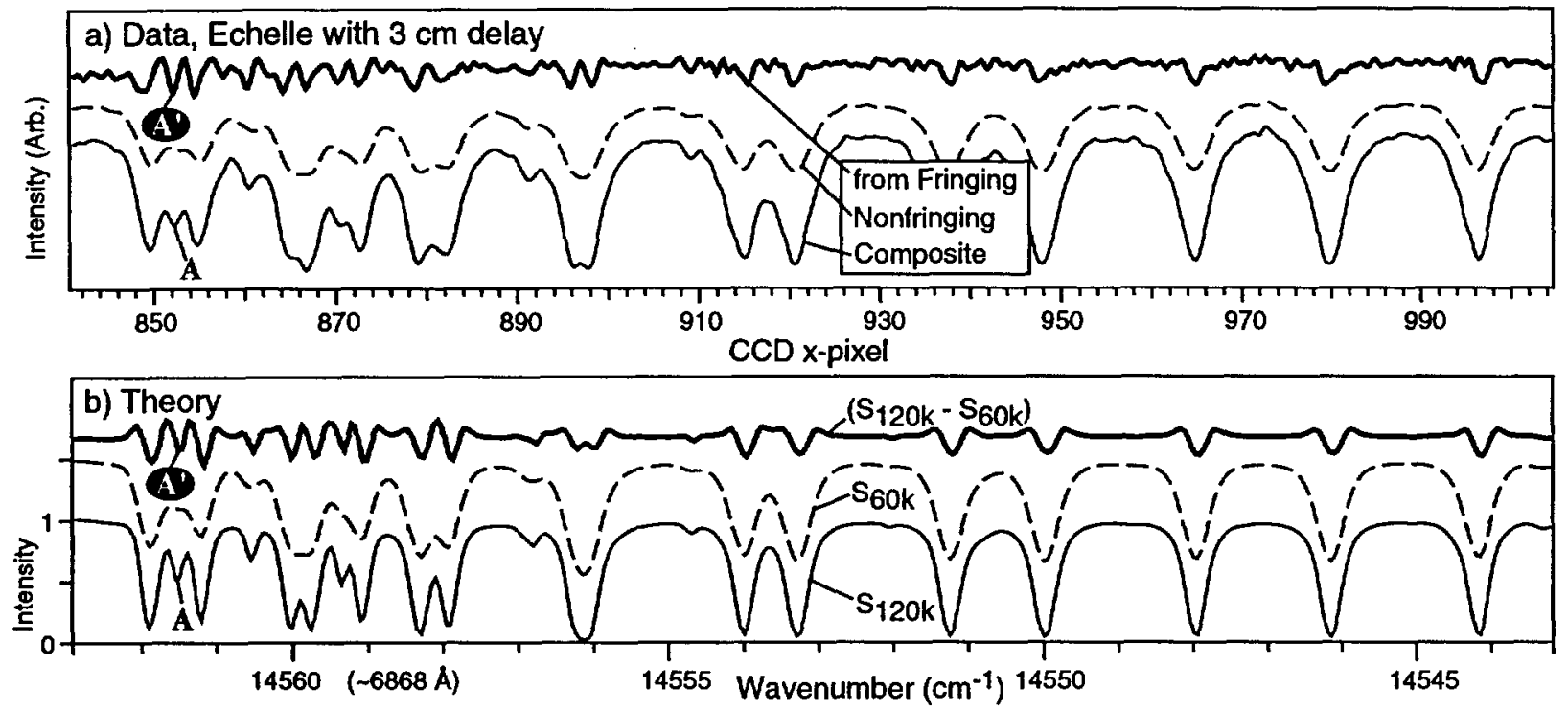

Figure 8. Demonstration of $\mathrm{a} \sim 2 \times \mathrm{EDI}$ resolution boosting effect on the Lick $\mathrm{R}=60 \mathrm{k}$ echelle grating spectrograph using $a \sim 3 \mathrm{~cm}$ delay interferometer and the telluric lines of the solar spectrum. The latter has narrow peaks, such as indicated by " $A$ ", that cannot be resolved at $R=60 \mathrm{k}$ without the interferometer (dashed curve), but are resolved in the composite EDI signal (thin curve). The bold curve is the fringing component of the data after the heterodyning effect is reversed numerically. Adding it to the nonfringing component produces the composite spectra having boosted resolution. The analysis is preliminary because the equalization step has not yet been applied. The theory spectra of panel b) were measured at $R \sim 500 \mathrm{k}$ by the Kitt Peak FTS and artificially blurred to $R=120 \mathrm{k}$ (thin) and $R=60 \mathrm{k}$ (dashed) resolutions. The resolution boosting analysis can be applied to each order of the echelle format to cover a bandwidth from infrared to ultraviolet.
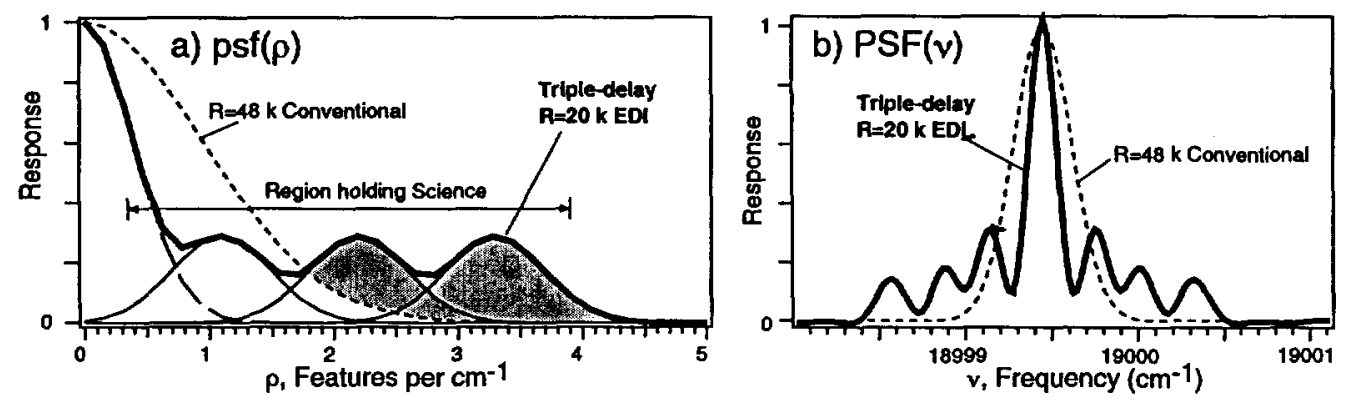

Figure 9. Instrument response (bold) of a triple-delay EDI in a) $\rho$-space and b) $\nu$-space. The delays are $1.1,2.2$ and $3.3 \mathrm{~cm}$. The height of heterodyning peaks (thin peaks) are reduced by $\sqrt{3}$ over the single delay height in Fig. 5 because photon noise depends on the square root of flux. The process of equalization would remove the bumpiness of the composite curve (bold) to form a response that is uniform over a wide range of $\rho$. The response is superior to the $\mathrm{R}=48 \mathrm{k}$ grating-alone for $\rho>1.8 \mathrm{~cm}$. b) The response in frequency space shows a much narrower peak than the $\mathrm{R}=48$ $k$ grating-alone curve (dashed). The ringing in the wings is not a practical concern because of the equalization process. 

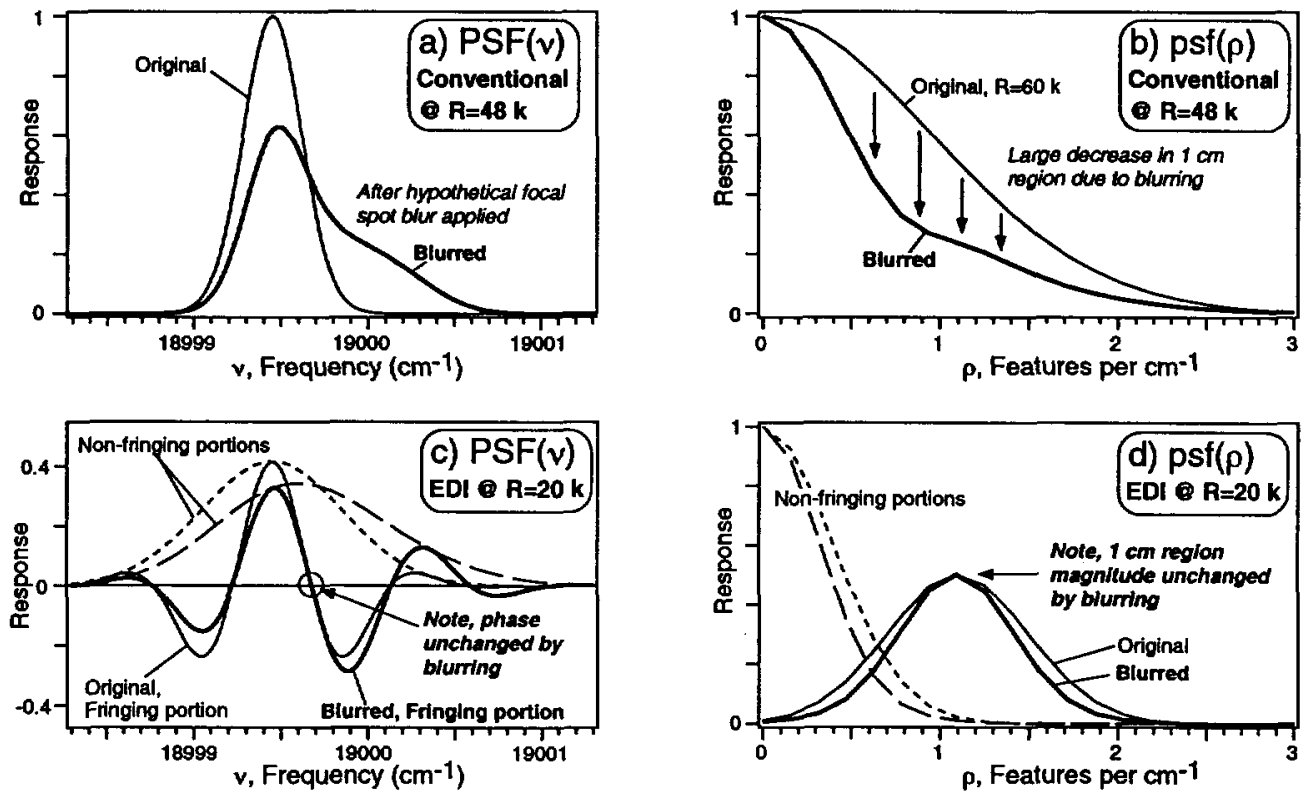

Figure 10. Demonstration of the robustness of the EDI technique to changes in shape of the external spectrograph PSF. a) The thin curve is the ideal $\mathrm{R}=48 \mathrm{k} P S F(\nu)$ of the external spectrograph. We suppose the focal spot on the CCD is blurred by some aberration, which we simulate by convolving the thin curve with a hypothetical blurring shape to form the bold curve. Note the large shift in the centroid which would produce a large Doppler error. b) The corresponding $p s f(\rho)$ are obtained from $P S F(\nu)$ by Fourier transform. Note that the blurring causes significant loss of information in the $\rho \sim 1 \mathrm{~cm}$ region (change from thin to bold curves). c) The original and blurred versions of the $P S F(\nu)$ for the EDI technique using $R=20 \mathrm{k}$. The fringing (thin) and nonfringing (dashed) components of the original PSF are shown. The hypothetical blurring function used in panel a) is convolved with the PSF to produce the blurred nonfringing PSF (long dashes) of the EDI technique, and from this the fringing PSF (bold). Note that the phase of the bold curve is not significantly effected by the blurring, since the bold curve is mostly formed from the interferometer behavior which is not effected by the focal spot blurring. Small phase shift means small Doppler error. d) Corresponding $p s f(\rho)$ for the EDI. The blurring causes the wings of the peaks to change, but not the tops of the peaks. Due to the heterodyning that places a peak at the $1.1 \mathrm{~cm}$ position, the $\rho \sim 1 \mathrm{~cm}$ region is not significantly effected by the blurring. 
The EDI is a hybrid instrument sharing advantages of both purely dispersive, and purely interferometric systems such as the Fourier Transform Spectrometer (FTS). As $M \rightarrow \infty$ the EDI regime approaches that of the FTS. The advantage of the EDI over the FTS is a much larger photon $\mathrm{s} / \mathrm{n}$ ratio (say 2 order of magnitude larger) to achieve scientifically useful resolutions. This increase in photon-limited $s / n$ is a consequence of adding external dispersion.

\subsection{Robustness to Changes in Grating PSF}

The EDI can be orders of magnitude more robust to errors caused by changes in the grating PSF, than the grating alone. This is due to the heterodyning effect. Changes in a grating $P S F(\nu)$ effect only the wings of the $p s f(\rho)$ and not the peak, and in the EDI the peak is shifted over the science region. Figure 10 gives a numerical demonstration of this robustness.

\section{ACKNOWLEDGMENTS}

The echelle data was taken with the assistance of Barry Welsh, Michael Feuerstein and Tim Erskine. Shauna Sallmen assisted in reduction of echelle data. Thanks to Tony Misch and Rem Stone for help in mounting the interferometer. We are grateful for the Lick Observatory and staff for use of their facility. Reference solar spectra were provided by Kitt Peak/NOAO. Work was partially supported by CalSpace/Lockheed, and NASA SARA research grants NAG5-9091 and NAG5-3051. This work was performed under the auspices of the U.S. Department of Energy by the University of California, Lawrence Livermore National Laboratory under contract No. W-7405-Eng-48.

\section{REFERENCES}

1. D. Erskine, "Single and double superimposing interferometer systems," US Patent 6,115,121, 2000.

2. D. Erskine, "Combined dispersive/interference spectroscopy for producing a vector spectrum," US Patent 6,351,307, 2002.

3. D. Erskine and J. Ge, "Novel interferometer spectrometer for sensitive stellar radial velocimetry," in Imaging the Universe in Three Dimensions: Astrophysics with Advanced Multi-Wavelength Imaging Devices, W. van Breugel and J. Bland-Hawthorn, eds., ASP 195, p. 501, 2000.

4. J. Ge, D. Erskine, and M. Rushford, "An externally dispersed interferometer for sensitive doppler extrasolar planet searches," PASP , 2002, accepted for publication.

5. J. Ge, "Fixed delay interferometry for doppler extrasolar planet detection," ApJ 571, pp. L165-168, 2002.

6. D. Erskine, "An externally dispersed interferometer prototype for sensitive radial velocimetry: Theory and demonstration on sunlight," PASP , 2002, submitted March 2002.

7. S. Vogt, "The Lick Observatory hamilton echelle spectrometer," PASP 99, p. 1214, 1987.

8. S. Vogt et al., "HIRES: The high resolution echelle spectrometer on the keck ten-meter telescope," Proc. SPIE 2198, p. 362, 1994.

9. R. Hilliard and G. Shepherd, J. Opt. Soc. Am. 56, p. 362, 1966.

10. N. Douglas, "Heterodyned holographic spectroscopy," PASP 109, p. 151, 1997.

11. S. Frandsen, N. Douglas, and H. Butcher, "An astronomical seismometer," $A \& A$ 279, p. 310, 1993.

12. J. Harlander, R. Reynold, and F. Roesler, "Spatial heterodyne spectroscopy for the exploration of diffuse interstellar emission lines at far-ultraviolet wavelengths," $A p J 396$, p. 730, 1992. 